\title{
Creatine supplementation post-exercise does not enhance training-induced adaptations in middle to older aged males
}

\author{
Matthew B. Cooke · Brian Brabham - Thomas W. Buford · Brian D. Shelmadine • \\ Matthew McPheeters • Geoffrey M. Hudson • Christos Stathis • Mike Greenwood • \\ Richard Kreider • Darryn S. Willoughby
}

Received: 10 November 2013 / Accepted: 28 February 2014 / Published online: 16 March 2014

(C) The Author(s) 2014. This article is published with open access at Springerlink.com

\begin{abstract}
Purpose The present study evaluated the effects of creatine monohydrate ( $\mathrm{CrM})$ consumption post-exercise on body composition and muscle strength in middle to older males following a 12-week resistance training program.

Methods In a double-blind, randomized trial, 20 males aged between 55 and 70 years were randomly assigned to consume either CrM-carbohydrate (CHO) [20 g days ${ }^{-1}$ $\mathrm{CrM}+5 \mathrm{~g} \mathrm{days}^{-1} \mathrm{CHO} \times 7$ days, then $0.1 \mathrm{~g} \mathrm{~kg}^{-1}$ $\mathrm{CrM}+5 \mathrm{~g} \mathrm{CHO}$ on training days (average dosage of $\sim 8.8 \mathrm{~g})$ ] or placebo $\mathrm{CHO}\left(20 \mathrm{~g} \mathrm{days}^{-1} \mathrm{CHO} \times 7\right.$ days,
\end{abstract}

Communicated by Michael Lindinger.

M. B. Cooke $(\bowtie) \cdot$ C. Stathis

College of Health and Biomedicine, Victoria University, PO Box

14428, Melbourne 8001, Australia

e-mail: matt.cooke@vu.edu.au

M. B. Cooke $\cdot$ B. D. Shelmadine $\cdot$ M. McPheeters ·

D. S. Willoughby

Department of Health, Human Performance and Recreation,

Baylor University, Waco, TX, USA

\section{B. Brabham}

Department of Exercise and Sport Science, University of Mary

Hardin-Baylor, Temple, TX, USA

T. W. Buford

Department of Aging and Geriatric Research, University

of Florida, Gainesville, FL, USA

G. M. Hudson

School of Public Health and Health Services, The George

Washington University, Washington, DC, USA

M. Greenwood · R. Kreider

Department of Health and Kinesiology, University of Texas

A\&M, College Station, TX, USA then $5 \mathrm{~g}$ CHO on training days) while participating in a high intensity resistance training program $[3$ sets $\times 10$ repetitions at $75 \%$ of 1 repetition maximum (1RM)], 3 days weeks ${ }^{-1}$ for 12 weeks. Following the initial 7-day "loading" phase, participants were instructed to ingest their supplement within 60 min post-exercise. Body composition and muscle strength measurements, blood collection and vastus lateralis muscle biopsy were completed at 0,4 , 8 and 12 weeks of the supplement and resistance training program.

Results A significant time effect was observed for 1RM bench press $(p=0.016)$, leg press $(p=0.012)$, body mass $(p=0.03)$, fat-free mass $(p=0.005)$ and total myofibrillar protein $(p=0.005)$. A trend for larger muscle fiber crosssectional area in the type II fibers compared to type I fibers was observed following the 12-week resistance training $(p=0.08)$. No supplement interaction effects were observed.

Conclusion Post-exercise ingestion of creatine monohydrate does not provide greater enhancement of body composition and muscle strength compared to resistance training alone in middle to older males.

Keywords Supplementation · Aging · Muscle · Hypertrophy $\cdot$ Creatine $\cdot$ Supplementation

$\begin{array}{ll}\text { Abbreviations } \\ \text { 1RM } & 1 \text { repetition maximum } \\ \text { ACSM } & \text { American College of Sports Medicine } \\ \text { CHO } & \text { Carbohydrate } \\ \text { CrM } & \text { Creatine monohydrate } \\ \text { CSA } & \text { Cross sectional area } \\ \text { DEXA } & \text { Dual energy X-ray absorptiometry } \\ \text { ELISA } & \text { Enzyme-linked immunoabsorbent assay } \\ \text { GH } & \text { Growth hormone }\end{array}$




$\begin{array}{ll}\text { ICC } & \text { Intraclass correlation coefficient } \\ \text { IGF-1 } & \text { Insulin-like growth factor 1 } \\ \text { MANOVA } & \text { Multivariate analysis of variance } \\ \text { RNA } & \text { Ribonucleic acid } \\ \text { SD } & \text { Standard deviation }\end{array}$

\section{Introduction}

Aging is associated with progressive loss of neuromuscular function that often leads to progressive disability and loss of independence. Sarcopenia is now a commonly used term to describe the loss of skeletal muscle mass and strength that occurs in concert with biological aging (Janssen et al. 2002; Bales and Ritchie 2002; Borst 2004). By the seventh and eighth decade of life, muscle strength can be reduced, on average, by approximately $20-40 \%$ for both men and women (Doherty 2003). Although age-associated decreases in strength per unit of muscle mass (i.e. muscle quality) may play a role, a large proportion of strength loss can be accounted for by decreased muscle mass (Doherty 2003). Multiple factors have been implicated in the development of sarcopenia and the associated impact on function. Loss of skeletal muscle fibers secondary to decreased numbers of motor neurons appears to be a major contributing influence, but other factors, including decreased physical activity, altered hormonal status, decreased total caloric and protein intake, inflammatory mediators, and factors leading to altered protein synthesis must also be considered (Buford et al. 2010).

In the past decade, strength training has been investigated extensively as a means of attenuating sarcopenia (Hunter et al. 2004; Candow and Chilibeck 2007). Indeed, high intensity resistance training is known to increase myofibrillar protein synthesis as well as muscle hypertrophy (Candow and Chilibeck 2007). However, literature suggests that despite training, muscle loss may still occur in older adults, indicating factors including nutrition may also play an important role in combating sarcopenia (Campbell and Leidy 2007).

One potentially efficacious method of leveraging nutrition to enhance the efficacy of resistance training is supplementation with creatine monohydrate $(\mathrm{CrM})$. In athletic populations, $\mathrm{CrM}$ has been extensively studied over the past 20 years, with its effects on high intensity, shortterm exercise well-documented (Rawson and Volek 2003; Williams and Branch 1998; Chrusch et al. 2001). To date, available evidence regarding the ergogenic effect of $\mathrm{CrM}$ supplementation in older adults is equivocal as some studies have reported improvement (Rawson and Wehnert 1999; Gotshalk et al. 2007; Brose and Parise 2003) or no change (Elliot et al. 2008; Bemben et al. 2010; Olsen et al. 2006) in various measures of body composition and muscle performance. In recent years, it has been suggested that the timing of nutritional supplementation in close proximity of an exercise bout maybe more important than absolute daily intake of the supplements (Cribb and Hayes 2006; Candow and Chilibeck 2008; Antonio and Ciccone 2013). Numerous studies have shown the benefit of protein timing on adaptations to resistance exercise in both young and older populations (Stark et al. 2012). However, research into the ergogenic potential of creatine ingestion in close proximity to exercise completion is limited. In young males and females, creatine ingestion $\left(0.2 \mathrm{~g} \mathrm{~kg}^{-1}\right.$, average dosage of $\sim 15.5 \mathrm{~g}$ ) following a single limb exercise for 6 weeks increased lean muscle mass (males only) and elbow flexor muscle thickness to a greater extent than placebo ingestion (Chilibeck et al. 2004). Conversely, creatine taken before and after exercise training at a lower dosage $\left(0.1 \mathrm{~g} \mathrm{~kg}^{-1}\right.$, average dosage of $\sim 5.7 \mathrm{~g}$ ) failed to provide any further increases in body mass, lean muscle mass and total muscle thickness when compared to placebo ingestion in older males (Candow et al. 2008). Recently, Antonio and Ciccone (2013) showed that consuming as little as $5 \mathrm{~g}$ of creatine post-exercise may affect the adaptive response to exercise in young male bodybuilders. However, the purpose of this study was to compare pre- vs. post-exercise consumption of creatine and thus a true control (i.e., no supplement) was not included (Antonio and Ciccone 2013). Subsequently, the effectiveness of creatine ingestion in close proximity of resistance exercise at a lower dosage is unclear.

While continuous CrM supplementation (acute or long term) appears to enhance resistance training-induced muscle adaptations in young and older individuals, the benefit of timing creatine ingestion relative to the resistance exercise session is unclear. Therefore, the primary purpose of this study was to determine the effect of post-exercise CrM consumption on body composition and muscle strength in middle to older males. We hypothesized that post-exercise CrM consumption concurrent with a resistance training program would result in greater training enhancement in upper and lower body muscle strength, lean muscle mass, muscle fiber cross-sectional area (CSA) when compared to $\mathrm{CHO}$ ingestion.

\section{Materials and methods}

\section{Participants}

Twenty-eight apparently healthy male participants between the ages of 55-70 years began this study. A total of eight participants were unable to complete the study for the following reasons: time commitment (4), uncomfortable with muscle biopsy procedure (2), and non-study related injury (2). A total of 20 participants (10 per group) completed 
all testing sessions and all 20 participants were used for subsequent analysis of all variables. Sample size was calculated using G Power software. Based on expected lean muscle mass changes (Chrusch et al. 2001) for the creatine and placebo groups of $55.85 \pm 1.65$ and $51.45 \pm 1.35 \mathrm{~kg}$, respectively, the calculated effect size is 2.92 . With an $\alpha$ of 0.05 , a sample size of 8 per group yields a power of 0.92 .

Participants were recreationally active (i.e. performed exercise 1-2 days weeks ${ }^{-1}$ ) but non-resistance trained (i.e. no regular resistance training for at least 1 year) Exclusionary criteria included smoking during previous year, use of nutritional supplements or supplemental androgens within the previous 6 months, current use of statins, recent history of cancer (within 2 years), neurological disease or cardiac arrhythmia interfering with physical function, peripheral vascular disease, congestive heart failure, complicated diabetes, chronic inflammatory disease, recent stroke (within 1 year), renal/kidney disease, and any absolute contraindications to exercise according to American College of Sports Medicine (ACSM) guidelines.

All eligible participants were asked to provide oral and written informed consent based on documents approved by the Institutional Review Board of Baylor University. All experimental procedures involved in the study conformed to the ethical consideration of the Helsinki Code. Verbal explanation of the purpose of the research, the protocol to be followed, and the experimental procedures to be used were given to the participants.

\section{Entry and familiarization session}

Participants expressing interest in participating in this study were interviewed on the phone and/or via email to determine whether they appeared to qualify to participate in this study. Participants believed to meet eligibility criteria were then invited to attend an entry/familiarization session. Participants were asked to bring signed physician approval of participation to the familiarization session. During this session, the potential participants signed informed consent statements and completed personal and medical history forms. Participants meeting entry criteria were familiarized to the study protocol, and the tests to be performed, via a verbal and written explanation outlining the study design. Participants were instructed to refrain from exercise for $48 \mathrm{~h}$ and fast for $8 \mathrm{~h}$ prior to testing.

\section{Experimental design}

Upon reporting to the lab for the exercise testing session, body mass, heart rate and blood pressure were determined prior to analyzing body composition using dual X-ray absorptiometry (DEXA) (Hologic, Waltham, MA). Following the DEXA scan, venous blood and skeletal muscle biopsy samples were obtained by standard/sterile procedures. Participants then performed upper body and lower body 1 repetition maximum (1RM) strength measurements. Participants were then matched on lower body 1RM strength, age and fat-free mass and randomly assigned to consume either $\mathrm{CrM}-\mathrm{CHO}$ or $\mathrm{CHO}$ placebo while participating in a high intensity resistance training program (3 sets $\times 10$ repetitions at $75 \%$ of $1 \mathrm{RM}), 3$ days weeks $^{-1}$ for 12 weeks. At 4 (T2), 8 (T3) and 12 (T4) weeks, participants returned to the lab to perform the same battery of tests as performed at baseline (T1) $48 \mathrm{~h}$ after the last workout. A total of four blood and muscle biopsy samples were taken during the study.

Muscle strength assessment

At $0,4,8$ and 12 weeks participants performed $1 \mathrm{RM}$ muscular strength tests using an isotonic $45^{\circ}$ leg press (Nebula Fitness, Inc., Versailles, $\mathrm{OH}$ ) and bench press (Nebula Fitness, Inc., Versailles, OH). Participants began the 1RM leg press and bench press test by first completing five to ten repetitions at approximately $50 \%$ of their previously established 1RM. Following a 2-min rest, three to five repetitions were performed at approximately $70 \%$ of their 1RM. From this time forward, the weight was increased gradually, until an official 1RM was reached. The rest period between each successful lift was 2 min. Test-retest reliability for performing these strength assessments on participants within our laboratory has demonstrated low mean coefficients of variation and high reliability for the bench press $(1.9 \%$, intraclass $r=0.94)$ and leg press $(0.7 \%$, intraclass $r=0.91$ ), respectively.

\section{Resistance training program}

Participants completed a partially supervised resistance training program. Partially supervised means that each participant was supervised for the first 2 weeks to ensure correct technique was being implemented. Participants were then monitored (i.e. correct technique used, correct weight lifted etc.) at the gym at 6,8 and 11 weeks. Participants utilized either local fitness centers of which they were a member or facilities on the Baylor University campus. Participants participated in a partially supervised resistance training program three times per week for the duration of the study. Participants completed resistance exercises similar to those utilized by Chrusch et al. (2001). Participants completed the bench press, lat pull-down, biceps curl, triceps press down, leg press, leg extension and leg curl. Three sets of 10 repetitions were completed for each exercise. An intensity of $75 \%$ of 1 RM was utilized during training. Upon successful completion of 3 sets of 10 repetitions, an increase of $5 \%$ of weight was utilized to 
ensure progressive overload during the study took place. A rest period of 1 min separated each set. Participants were asked to keep training logs detailing amount of weight utilized, number of repetitions completed and number of sets completed.

\section{Nutritional supplementation}

Participants were randomly assigned to receive, in a doubleblind manner, either $20 \mathrm{~g}$ of CrM (Alschem, Degussa Inc., Atlanta, GA) combined with $5 \mathrm{~g}$ of glucose (DGC AST Sport Science, Golden, CO) for 7 days followed by $0.1 \mathrm{~g} \mathrm{~kg}^{-1}$ (average dosage of $\sim 8.8 \mathrm{~g}$ ) of CrM with $5 \mathrm{~g}$ of glucose on training days or $20 \mathrm{~g}$ of glucose only for 7 days followed by $5 \mathrm{~g}$ of glucose on training days. Each supplement dosage was provided in small identical plastic bags. Supplement was given to participants in a 4-week block period and thus dosage could be adjusted following body-weight analysis. Each supplement was similar in color and texture. A small shaker bottle was provided to participants with instructions on how to mix the supplement in about $500 \mathrm{ml}$ of water. Participants receiving CrM completed a 7-day loading period with $20 \mathrm{~g}$ of CrM being ingested daily in $4 \times 5 \mathrm{~g}$ increments (i.e. $9 \mathrm{am}$, 12,3 and $6 \mathrm{pm}$ ). Participants in both the experimental group (CrM-CHO) and the placebo control group ( $\mathrm{CHO}$ ) began the designed supplementation protocol immediately after baseline testing which took place on Day 0. Following the 7 day loading phase, participants were instructed to ingest either the supplement or placebo within 60 min following the resistance exercise session for the duration of the 12-week study period. Justification of the supplementation protocol is threefold: First, a lower dosage of $0.07-0.1 \mathrm{~g} \mathrm{~kg}^{-1}$ (approximately $5-8 \mathrm{~g}$ days $^{-1}$ ) results in minor adverse events (i.e., loose stools, muscle cramping, and muscle pulls/strains) (Chrusch et al. 2001; Candow et al. 2008). Second, a dosage as little as $5 \mathrm{~g}$ days $^{-1}$, albeit continuous, has shown to be effective at increasing lean muscle mass and strength in older males (Brose and Parise 2003; Chrusch et al. 2001). And finally, a single $5 \mathrm{~g}$ oral dose of CrM in healthy adults results in a peak plasma creatine level of approximately $120 \mathrm{mg} / \mathrm{l}$ at 1-2 $\mathrm{h}$ post-ingestion (i.e., during that post-exercise window) (Jager et al. 2007). Compliance monitoring was accomplished by having participants return empty containers of the supplement or placebo at each testing session. Compliance to the supplementation protocol was monitored by supplement $\operatorname{logs}$ and verbal confirmation. Compliance was also verified through the administration and collection of questionnaires from each participant regarding any noticeable side-effects.

\section{Tissue and blood sampling}

Skeletal muscle biopsies were collected accordingly to previously published procedures (Cooke et al. 2011). Briefly, percutaneous muscle biopsies were obtained using the Bergstroem technique under local anesthesia of $1 \%$ Xylocaine from middle portion of the vastus lateralis muscle of the dominant limb at the midpoint between the patella and the greater trochanter of the femur at a depth between 2 and $3 \mathrm{~cm}$. Muscle samples were obtained by standard/sterile procedures by personnel who were experienced in performing the procedure. After removal, the tissue samples were immediately frozen in liquid nitrogen and then stored at $-80{ }^{\circ} \mathrm{C}$ for future analyses.

Participants were required to fast overnight prior to donating approximately $20 \mathrm{ml}$ of venous blood from the antecubital vein using standard phlebotomy procedures. Blood was collected into serum separation tubes and centrifuged at $1,100 \mathrm{~g}$ for $15 \mathrm{~min}$. Serum was separated and stored at $-80^{\circ} \mathrm{C}$ in polypropylene tubes for later analysis.

Serum insulin-like growth factor 1 and testosterone

Serum samples were analyzed in duplicate for free/bioactive IGF-1 (Diagnostic Systems Laboratories, Webster, TX), and free testosterone (Alpha Diagnostic International, San Antonio, TX) using an ELISA (enzyme linked immunoabsorbent assay). For IGF-1, assay sensitivity is $0.06 \mathrm{ng} /$ $\mathrm{ml}$, and does not cross-react with albumins or GH (growth hormone) binding proteins. For testosterone, sensitivity is $0.17 \mathrm{pg} / \mathrm{ml}$. For IGF-1 and testosterone the subsequent absorbances, which were directly proportional to the concentration of analyte in the sample, were measured at a wavelength of $450 \mathrm{~nm}$ using a microplate reader (Wallac Victor 1420, Perkin Elmer, Boston MA). A set of standards of known concentrations for IGF-1 and testosterone were utilized to construct standard curves by plotting the net absorbance values of the standards against their respective protein concentrations. By applying a four-part parameter curve using MikroWin microplate data reduction software (Microtek Lab Systems, Germany), the free IGF-1 and free testosterone concentrations in the serum samples were calculated. The overall intra-assay percent coefficient of variation was 4.9 and $3.7 \%$ for IGF-1 and testosterone, respectively.

Fiber type and cross-sectional area

Muscle tissue used for determination of muscle fiber type and CSA were taken at 0 and 12 weeks. Tissue obtained from the biopsy procedure was mounted using OCT medium and snap frozen in isopentane, pre-cooled in liquid nitrogen, and stored at $-80{ }^{\circ} \mathrm{C}$ for histochemical analysis to classify muscle fiber types into fast (II) and slow (I) on the basis of the stability of their ATPase activity, as previously described (Cribb and Hayes 2006). Fiber type CSA was determined from sections containing a mean of 210 
(range 130-400) fibers. Samples were measured on two separate occasions for day-to-day reproducibility. ICC (intraclass correlation coefficient) and SD (standard deviation) for fiber type distribution were type I $r=0.81$, SD $1.6 \%$ and type II $r=0.93$, SD $1.4 \%$.

\section{Myofibrillar protein content}

Total cellular RNA (ribonucleic acid) was extracted from biopsy samples with a monophasic solution of phenol and guanidine isothiocyanate contained within the TRI-reagent (Sigma Chemical Co., St. Louis, MO), and then isolated with $100 \%$ isopropanol. The interphase was removed and total (soluble + insoluble) muscle protein was then isolated from the organic phase with $100 \%$ isopropanol and washed with a $0.3 \mathrm{M}$ guanidine $\mathrm{HCl} / 95 \%$ ethanol solution. Myofibrillar (soluble) protein was further isolated with repeated incubations in $0.1 \%$ SDS at $50{ }^{\circ} \mathrm{C}$ and separated by centrifugation. Total myofibrillar protein content was determined spectrophotometrically based on the Bradford method at a wavelength of $595 \mathrm{~nm}$ (Bradford 1976). A standard curve was generated $(R=0.98, p=0.001)$ using bovine serum albumin (Bio-Rad, Hercules, CA), and total myofibrillar protein content was expressed relative to muscle wet-weight.

\section{Dietary records}

Participants were required to keep dietary records for 7 days prior to the exercise testing sessions. The 7-day dietary records were evaluated with the ESHA Food Processor 8.6 (ESHA Research, Salem, OR) dietary assessment software program to determine the average daily macronutrient consumption of fat, carbohydrate and protein, and consumption of antioxidant-related micronutrients vitamin $\mathrm{A}$, vitamin $\mathrm{C}$, vitamin $\mathrm{E}$ and selenium in the diet.

\section{Statistical analysis}

Data was analyzed by utilizing separate $2 \times 4$ [group $(\mathrm{CrM}-\mathrm{CHO}, \mathrm{CHO})] \times$ test (week 0, week 4 , week 8, week 12) mixed design factorial multivariate analysis of variance (MANOVA). MANOVAs were analyzed for this study based on dependent variables that are likely to be related to one another. In addition, the use of a MANOVA analysis prevents the potential for the increasing of type I error rate that might result with the use of repeated univariate procedures. ANOVA on each dependent variable was conducted as a follow-up test to any significant MANOVA. To control for alpha inflation of the ANOVA, the Bonferroni test was utilized as a follow-up test. Post-hoc tests of any interaction effects demonstrated in the ANOVA were investigated via an independent samples $t$ test. In addition to reporting probability values, an index of effect size was reported to reflect the magnitude of the observed effect. The index of effect size utilized was the partial Eta squared $\left(\eta^{2}\right)$, which estimates the proportion of variance in the dependent variable that can be explained by the independent variable. Partial Eta squared effect sizes were determined to be: weak $=0.17$, medium $=0.24$, strong $=0.51$, very strong $\geq 0.70$ as previously described by O'Connor, et al. All statistical procedures were performed using SPSS 20.0 software (Chicago, IL) and an alpha probability level of $<0.05$ was adopted throughout. In addition, for all statistical analyses not meeting the sphericity assumption for the within-participant analyses, a Huynh-Feldt correction factor was applied to the degrees of freedom to adjust (increase) the critical $F$ value to a level that would prevent the likelihood of committing a type I error.

\section{Results}

Participants

At baseline, there were no significant differences between groups with regards to height $(\mathrm{cm})(\mathrm{CrM}-\mathrm{CHO}: 177.3 \pm 7.4$ vs. CHO: $175.1 \pm 5.0, p=0.451)$, body weight $(\mathrm{kg})(\mathrm{CrM}$ CHO: $88.2 \pm 12.3$ vs. CHO: $94.4 \pm 15.3, p=0.332)$, and age (years) (CrM-CHO: $61.4 \pm 5.0$ vs. CHO: $60.7 \pm 5.4$, $p=0.762$ ). A Shapiro-Wilk test was used to test for the normality of data and given non-significance was found; we were able to reject the alternative hypothesis and conclude that the data came from a normal distribution.

Nutritional intake analysis

All participants were instructed to consume their usual diet during the 12-week course of the study. Dietary analysis does not include information related to the supplement ingested. However, monitoring of supplement logs and verbal confirmation of supplement compliance (data not shown) indicated that supplementation compliance was $100 \%$. Independent sample $t$ tests were utilized to analyze all relevant dietary variables prior to commencement of supplementation and the resistance training program. Table 1 illustrates that at baseline (T1), there were no significant differences observed between groups for total daily caloric intake, macronutrient intake of protein, or carbohydrate. Following supplementation, a multivariate analysis revealed a strong trend towards significance for time [Wilk's Lambda $=0.057, F(4,12)=5.514, p=0.056$, effect size $\left(\eta^{2}\right)=0.943$, power $\left.=0.632\right]$. Univariate analysis detected a significant time effect for dietary fat intake $\left(p=0.025, \eta^{2}=0.189\right.$, power $\left.=0.727\right)$, with subsequent post-hoc analysis revealing significant increases in 
Table 1 Comparison of nutritional intake variables between CrM$\mathrm{CHO}$ and $\mathrm{CHO}$ Groups

\begin{tabular}{|c|c|c|c|c|}
\hline Variable & Time & CrM-CHO & $\mathrm{CHO}$ & Significance \\
\hline \multirow{4}{*}{$\begin{array}{l}\text { Energy intake } \\
\left(\text { kcal days }^{-1}\right)\end{array}$} & $\mathrm{T} 1$ & $2,127 \pm 423$ & $1,889 \pm 374$ & Group $=0.883$ \\
\hline & $\mathrm{T} 2$ & $1,834 \pm 681$ & $2,006 \pm 600$ & Time $=0.142$ \\
\hline & $\mathrm{T} 3$ & $1,939 \pm 454$ & $1,834 \pm 528$ & $G \times T=0.167$ \\
\hline & $\mathrm{T} 4$ & $2,024 \pm 490$ & $2,432 \pm 680$ & \\
\hline \multirow{4}{*}{$\begin{array}{r}\text { CHO intake } \\
\left(\mathrm{g} \text { days }^{-1}\right)\end{array}$} & $\mathrm{T} 1$ & $232 \pm 61$ & $208 \pm 60$ & Group $=0.901$ \\
\hline & $\mathrm{T} 2$ & $223 \pm 120$ & $241 \pm 119$ & Time $=0.885$ \\
\hline & T3 & $237 \pm 53$ & $211 \pm 97$ & $G \times T=0.603$ \\
\hline & $\mathrm{T} 4$ & $228 \pm 83$ & $241 \pm 139$ & \\
\hline \multirow{4}{*}{$\begin{array}{l}\text { Protein intake } \\
\left(\mathrm{g} \mathrm{days}^{-1}\right)\end{array}$} & $\mathrm{T} 1$ & $98 \pm 34$ & $83 \pm 18$ & Group $=0.829$ \\
\hline & $\mathrm{T} 2$ & $84 \pm 28$ & $78 \pm 8$ & Time $=0.161$ \\
\hline & $\mathrm{T} 3$ & $75 \pm 15$ & $73 \pm 13$ & $G \times T=0.449$ \\
\hline & $\mathrm{T} 4$ & $91 \pm 19$ & $108 \pm 74$ & \\
\hline \multirow{4}{*}{$\begin{array}{l}\text { Fat intake } \\
\left.\qquad \text { ( days }^{-1}\right)\end{array}$} & $\mathrm{T} 1$ & $95 \pm 36$ & $81 \pm 24$ & Group $=0.773$ \\
\hline & $\mathrm{T} 2$ & $68 \pm 25$ & $84 \pm 29$ & Time $=0.056$ \\
\hline & $\mathrm{T} 3$ & $76 \pm 31$ & $80 \pm 25$ & $G \times T=0.207$ \\
\hline & $\mathrm{T} 4$ & $83 \pm 28$ & \multicolumn{2}{|c|}{$116 \pm 32^{\dagger, *, \&}$} \\
\hline
\end{tabular}

Data are presented as mean \pm standard deviation

$\dagger$ Significantly different from T2 $(p<0.05)$

$¥$ Significantly different from T3 $(p<0.05)$

$\S$ Significantly different between groups $(p<0.05)$

dietary fat intake from T2 to T4 $(p=0.009)$ and T3-T4 $(p=0.013)$. In addition, dietary fat intake was significantly higher in the $\mathrm{CHO}$ group compared to the $\mathrm{CrM}-\mathrm{CHO}$ group at T4 $(p=0.04)$. These results indicate that while both groups increased their dietary fat intake from $\mathrm{T} 2$ to $\mathrm{T} 4$, the $\mathrm{CHO}$ group was ingesting significantly more dietary fat compared to the CrM-CHO group at T4. No other significant main effects for time, group, or group by time interactions were detected for dietary protein and carbohydrate intake.

Total lifting volume analysis

All participants were instructed to record, for each exercise, the number of repetitions and weight lifted at each training session. Total lifting volume for each group reflects the total number of repetitions multiplied by the total weight lifted performed by each participant for each exercise (4 weeks: CrM-CHO: $118,780 \pm 25,485 \mathrm{~kg}$, CHO: $129,500 \pm 33,137 \mathrm{kgs}, p=0.500 ; 8$ weeks: CrM-CHO: $\quad 130,060 \pm 38,313$ kgs, CHO: $147,000 \pm 40,384 \mathrm{kgs}, p=0.422 ; 12$ weeks: CrM-CHO: $142,220 \pm 55,236 \mathrm{kgs}$, CHO: $156,630 \pm 41,715 \mathrm{kgs}$, $p=0.331)$. Independent sample $t$ tests were utilized to analyze the total lifting volume at baseline, and repeated measures ANOVA was utilized to assess changes in total lifting volume over time. A statistical trend for time
$\left[F(2,12)=3.414, p=0.067\right.$, effect $\left.\operatorname{size}\left(\eta^{2}\right)=0.363\right]$ was detected indicating both groups increased their training output following 12 weeks of resistance training.

Muscle strength analysis

Data for 1RM bench press and leg press are represented in Figs. 1 and 2. Mulitivariate analysis indicated a significant time effect [Wilk's lambda $=0.289$, $F(6,13)=5.336, p=0.006, \eta^{2}=0.71$, power $\left.=0.941\right]$ with no significant group or group $\times$ time interactions. Univariate analysis revealed a significant time effect for $1 \mathrm{RM}$ bench press $[F(3,16)=0.016, p=0.016$, effect size $\left.\left(\eta^{2}\right)=0.468\right]$, with subsequent pairwise analysis displaying significant increases from baseline (T1) to T3 $(p=0.022)$, and T4 $(p=0.003)$, and T2-T3 $(p=0.022)$, and T4 $(p=0.002)$. Similarly, univariate analysis revealed a significant time effect for $1 \mathrm{RM}$ leg press $\left[F(3,16)=10.543, p=0.012\right.$, effect size $\left.\left(\eta^{2}\right)=0.664\right]$, with subsequent pairwise analysis revealing a significant increase from baseline (T1) to T2 $(p<0.05)$, T3 $(p<0.01)$ and T4 $(p<0.05)$.

\section{Body composition analysis}

Data for body mass $(\mathrm{kg})$, body fat (\%), fat-free mass $(\mathrm{kg})$ and fat mass $(\mathrm{kg})$ are presented in Table 2. Multivariate analysis revealed a significant effect for time (Wilks's Lambda $=0.046, \mathrm{~F}(4,15)=12.108, p=0.001$, $\eta^{2}=0.954$, power $\left.=0.998\right)$ and a trend towards significance for group effect $(p=0.078)$. No group $\times$ time interaction was found. Univariate analysis indicated a significant time effect for body mass $[F(3,16), p=0.030$, $\left.\eta^{2}=0.419\right]$ with subsequent pairwise analysis revealing significant increases in body mass between baseline (T1) and T2 $(p=0.008), \mathrm{T} 3(p=0.005)$, and T4 $(p=0.003)$ indicating both $\mathrm{CrM}-\mathrm{CHO}$, and $\mathrm{CHO}$ significantly increased body mass following 12 weeks of resistance training. A strong statistical trend for group was detected for body fat $\%(p=0.058)$, indicating higher body fat $\%$ in the $\mathrm{CHO}$ group compared to the $\mathrm{CrM}-\mathrm{CHO}$ group throughout the study period. A significant time effect was detected for fat-free mass $\left(p=0.005, \eta^{2}=0.212\right.$, power $=0.884$ ) with subsequent pairwise analysis revealing significant increases between baseline (T1) and T2 $(p=0.004)$, T3 $(p=0.004)$, and T4 $(p=0.018)$, indicating both $\mathrm{CrM}-\mathrm{CHO}$, and $\mathrm{CHO}$ significantly increased fatfree mass following 12 weeks of resistance training. Similar to body fat $\%$, a trend towards significance for group was detected for fat mass $(p=0.075)$, indicating higher body fat $\%$ in the $\mathrm{CHO}$ group compared to the CrM-CHO group during the study period. 
Fig. 1 Time course of 1RM bench press. Data (mean \pm SD) represents $1 \mathrm{RM}$ bench press for both CrM-CHO and $\mathrm{CHO}$ groups, including the mean average for both groups combined, following 12 weeks of resistance training. Asterisk significantly different from baseline (T1) $(p<0.05)$. Dagger symbol significantly different from T2 $(p<0.05)$. Please note that symbols used are a reflection of pairwise analysis for time effects and thus differences between mean averages for both groups combined (mean average line in figure) at each time point

Fig. 2 Time course of 1RM leg press. Data (mean $\pm \mathrm{SD}$ ) represents $1 \mathrm{RM}$ leg press for both $\mathrm{CrM}-\mathrm{CHO}$ and $\mathrm{CHO}$ groups, including the mean average for both groups combined, following 12 weeks of resistance training. Asterisk significantly different from baseline (T1) $(p<0.05)$. Please note that symbols used are a reflection of pairwise analysis for time effects and thus differences between mean averages for both groups combined (mean average line in figure) at each time point
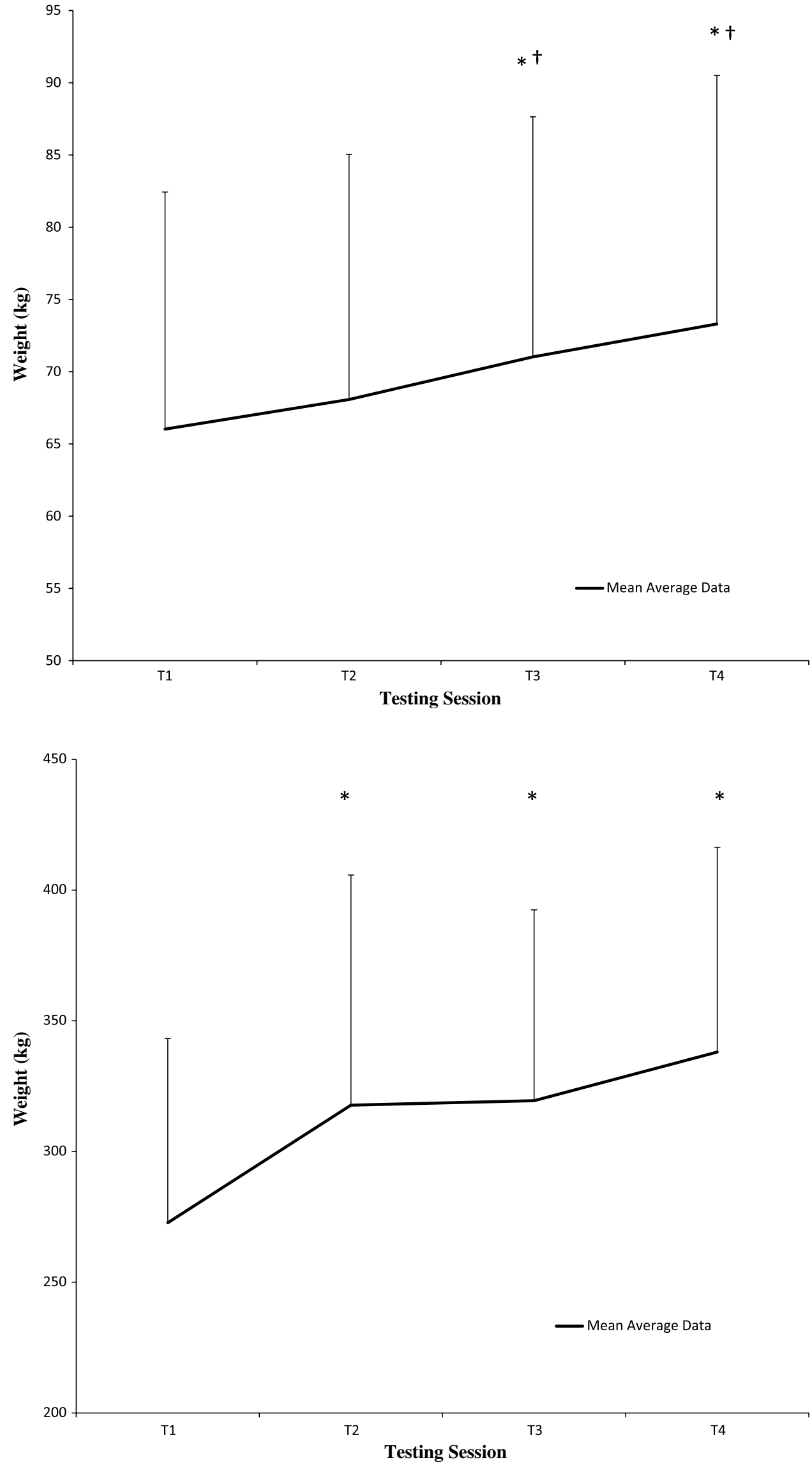
Table 2 Body composition for $\mathrm{CrM}-\mathrm{CHO}$ and $\mathrm{CHO}$ groups

\begin{tabular}{|c|c|c|c|c|c|}
\hline Variable & $\mathrm{T} 1$ & $\mathrm{~T} 2$ & $\mathrm{~T} 3$ & $\mathrm{~T} 4$ & Significance \\
\hline Body mass (Kg) & & & & & Group $=0.093$ \\
\hline $\mathrm{CrM}-\mathrm{CHO}$ & $88.2 \pm 12.4$ & $89.3 \pm 11.9 *$ & $89.8 \pm 12 *$ & $89.9 \pm 11.8^{*}$ & Time $=0.030$ \\
\hline $\mathrm{CHO}$ & $94.4 \pm 15.3$ & $94.9 \pm 15.1 *$ & $94.8 \pm 15.3^{*}$ & $95.1 \pm 15.5^{*}$ & $G \times T=0.638$ \\
\hline Body fat (\%) & & & & & Group $=0.058$ \\
\hline $\mathrm{CrM}-\mathrm{CHO}$ & $26.7 \pm 3.5$ & $26.1 \pm 3.4$ & $25.6 \pm 2.6$ & $26.5 \pm 3.8$ & Time $=0.232$ \\
\hline $\mathrm{CHO}$ & $29.8 \pm 4.3$ & $29.3 \pm 3.8$ & $29.6 \pm 4.2$ & $29.8 \pm 4.9$ & $G \times T=0.538$ \\
\hline Fat mass (Kg) & & & & & Group $=0.075$ \\
\hline $\mathrm{CrM}-\mathrm{CHO}$ & $21.6 \pm 4.8$ & $21.5 \pm 4.6$ & $21.3 \pm 5.0$ & $21.0 \pm 4.3$ & Time $=0.103$ \\
\hline $\mathrm{CHO}$ & $25.8 \pm 5.3$ & $25.6 \pm 5.0$ & $26.0 \pm 5.9$ & $26.2 \pm 6.4$ & $G \times T=0.289$ \\
\hline Fat-free mass $(\mathrm{Kg})$ & & & & & Group $=0.516$ \\
\hline CrM-CHO & $56.6 \pm 7.1$ & $58.3 \pm 7.2 *$ & $59.1 \pm 7.4^{*}$ & $58.6 \pm 7.9^{*}$ & Time $=0.005$ \\
\hline $\mathrm{CHO}$ & $58.9 \pm 11.2$ & $60.1 \pm 11.1^{*}$ & $59.6 \pm 10.6^{*}$ & $59.4 \pm 10.7 *$ & $G \times T=0.769$ \\
\hline
\end{tabular}

Data are presented as mean \pm standard deviation

* Significantly different from baseline (T1) $(p<0.05)$

Table 3 Serum variables for CrM-CHO and CHO groups

\begin{tabular}{llllll}
\hline Variable & T1 & T2 & T3 & T4 & Significance \\
\hline IGF-1 (pg/ml) & & & & $505 \pm 304$ & Group $=0.093$ \\
CrM-CHO & $510 \pm 300$ & $424 \pm 322$ & $532 \pm 310$ & $283 \pm 312$ & $G \times T=0.638$ \\
CHO & $280 \pm 300$ & $280 \pm 230$ & $345 \pm 245$ & & Time $=0.261$ \\
Testosterone (pg/ml) & & & $123 \pm 89$ & Group $=0.516$ \\
CrM-CHO & $114 \pm 94$ & $116 \pm 84$ & $126 \pm 69$ & $148 \pm 126$ & Time $=0.531$ \\
CHO & $159 \pm 110$ & $158 \pm 146$ & $G \times 0.769$ \\
\hline
\end{tabular}

Data are presented as mean \pm standard deviation

Serum analysis

Data for serum IGF-1 and free testosterone are represented in Table 3. Multivariate analysis indicated a trend for time [Wilk's Lambda $=0.276, F(9,10), p=0.056$, $\eta^{2}=0.724$, power $\left.=0.703\right]$. No significant time, group or group $\times$ time interaction was observed.

Skeletal muscle analysis

Data for total myofibrillar protein concentration and muscle fiber CSA are presented in Table 4. A repeated measures ANOVA revealed a significant time effect for total myofibrillar protein content $[F(3,15)=3.509, p=0.042$, effect size $\left(\eta^{2}\right)=0.412$ ] with no significant group or group $\times$ time interactions. Subsequent pairwise analysis showed a significantly higher total myofibrillar protein concentration at T4 compared to baseline $(p=0.005)$. Student's $t$ test revealed a trend $(p=0.08)$ for an increase in size of type II muscle fiber CSA following 12-week resistance training. No significant differences were observed between type I and II CSA or following creatine supplementation.

\section{Discussion}

The primary purpose of this study was to determine if the consumption of creatine in the hour window post-exercise would stimulate greater training-induced increases in lean muscle mass, muscle fiber CSA and myofibrillar protein content concomitant with upper and lower body muscle strength gains compared to $\mathrm{CHO}$ placebo ingestion. In the current study, 12 weeks of resistance training increased body mass, fat-free mass and both upper body and lower body strength. In addition, significantly higher levels of myofibrillar protein concentration and larger muscle fiber CSA, albeit not significant, were observed following the 12-week resistance training program. Despite these improvements, ingesting creatine post-exercise did not enhance the observed resistance training-induced changes in body composition and/or muscle strength. 
Table 4 Muscle variables for CrM-CHO and CHO groups

\begin{tabular}{|c|c|c|c|c|c|}
\hline Variable & $\mathrm{T} 1$ & $\mathrm{~T} 2$ & $\mathrm{~T} 3$ & $\mathrm{~T} 4$ & Significance \\
\hline \multicolumn{6}{|c|}{ Fiber CSA $\left(\mu \mathrm{m}^{2} 10^{-3}\right)$} \\
\hline CrM-CHO type I & $3.1 \pm 0.6$ & & & $4.8 \pm 1.1$ & \\
\hline CHO type I & $3.8 \pm 1.5$ & & & $4.7 \pm 0.9$ & \\
\hline CrM-CHO type II & $2.9 \pm 1.1$ & & & $4.0 \pm 0.6$ & \\
\hline CHO type II & $3.2 \pm 0.6$ & & & $4.0 \pm 0.9$ & \\
\hline \multicolumn{5}{|c|}{ Total myofibrillar protein content ( $\mu \mathrm{g} / \mathrm{mg}$ muscle) } & Group $=0.316$ \\
\hline $\mathrm{CrM}-\mathrm{CHO}$ & $7.9 \pm 4.0$ & $10.5 \pm 8.7$ & $10.1 \pm 1.9$ & $12.0 \pm 3.4^{*}$ & Time $=0.042$ \\
\hline $\mathrm{CHO}$ & $8.5 \pm 2.2$ & $8.1 \pm 2.6$ & $11.7 \pm 7.7$ & $11.1 \pm 4.4$ & $G \times T=0.669$ \\
\hline
\end{tabular}

Data are presented as mean \pm standard deviation

* Significantly different from baseline (T1) $(p<0.05)$

In young and older populations, greater gains in body and lean muscle mass and muscular strength have been observed following a standard creatine supplementation protocol in conjunction with a resistance training program compared to supplementation or resistance training alone (Becque and Lochman 2000; Bermon et al. 1998; Chrusch et al. 2001; Kreider 2003; Willoughby and Rosene 2001). Participants typically consume $0.3 \mathrm{~g} \mathrm{~kg}^{-1}$ or approximately 20-25 $\mathrm{g} \mathrm{days}^{-1}$ of CrM for 5-7 days ("loading phase") and $0.07 \mathrm{~g} \mathrm{~kg}^{-1}$ or approximately $2-5 \mathrm{~g} \mathrm{days}^{-1}$ of $\mathrm{CrM}$ thereafter ("maintenance phase"). Although this protocol has been shown to be effective, others have excluded the loading phase and only used a continuous low dosage (i.e., 5-7.7 $\mathrm{g}$ days $^{-1}$ ) and were able to increase lean muscle mass and muscular performance over placebo-supplemented groups (Brose and Parise 2003; Burke et al. 2000).

In the current study following a standard "loading" protocol, CrM ingestion of $\sim 8.8 \mathrm{~g}$ post-exercise was unable to enhance body composition (i.e. gains in fat-free mass, loss of body fat), muscle strength, total myofibrillar protein content and/or individual muscle fiber CSA following a 12-week resistance training program. These observations are in agreement with others who have used a lower dosage (3-5.7 g) before and/or after exercise and failed to show any benefit on exercise-induced adaptations to exercise (Candow et al. 2008; Eijnde et al. 2003). Eijnde et al. (2003) instructed their participants to ingest $3 \mathrm{~g}$ of creatine (of the $5 \mathrm{~g} \mathrm{days}^{-1}$ ) post-exercise for a period of 24 weeks; where as Candow et al. (2008) ingested approximately $2.85 \mathrm{~g}$ of creatine (of the approximate $8 \mathrm{~g}$ days $^{-1}$ ) before and after exercise for a period of 10 weeks. Interestingly, when protein $\left(\sim 24 \mathrm{~g}\right.$ days $\left.^{-1}\right)$ was combined with the low creatine dosage, greater gains were seen in lean muscle mass and strength compared to placebo group (Candow et al. 2008). However, given the well established effects of protein/amino acid supplementation on muscle protein stimulation, the observed results are most likely due to protein supplementation and not the creatine per se.
Contrary to our findings, Chilibeck et al. (2004) demonstrated greater increases in elbow flexor muscle thickness and lean mass tissue (males only) when creatine was ingested after a single limb training session twice a week for 6 weeks. It is not readily apparent as to why our hypothesis was not supported. It is clear that the dosage used by Chilibeck et al. (2004) was higher and the duration was shorter compared to the current study ( 15.5 vs. $\sim 8.8 \mathrm{~g}, 6$ vs. 12 weeks) and thus could explain why the current study didn't show any significant benefit from the post-exercise creatine supplementation. However, when you average the dosage intake over the entire week, the difference seems to be minor $\left(\sim 4.5\right.$ vs. $\left.\sim 3.8 \mathrm{~g}^{\text {days }}{ }^{-1}\right)$. We decided to use a lower dosage and longer supplement duration because others (Chrusch et al. 2001; Brose and Parise 2003) have demonstrated that following a typical "loading" protocol and/ or continuous low dosage $\left(5 \mathrm{~g}\right.$ days $\left.^{-1}\right)$ supplementation period combined with resistance exercise, enhancement of body composition and improvements in muscular performance are typically observed. Although not measured in the study by Chrusch et al. (2001), the level of intramuscular creatine has been shown to influence the performance (Snow and McKenna 1998; Casey and Constantin-Teodosiu 1996); with a positive effect following CrM supplementation normally associated with large increases in intramuscular creatine following supplementation. Thus, we wanted to determine if post-exercise consumption (at a low dosage) following a typical "loading" protocol would be effective.

Therefore, the question is: Does a certain level of intramuscular creatine need to be achieved (i.e., via a typical "loading" protocol and/or continuous ingestion) to stimulate muscle growth or can acute muscle uptake of creatine (i.e., following pre- and/or post-exercise ingestion) have a similar effect? A limitation in our study and Chilibeck et al. (2004) is that total intramuscular CrM concentrations were not measured. Unlike Chilibeck et al. (2004), we performed a standard loading protocol at the beginning and can only speculate that total intramuscular creatine levels 
were increased and maintained throughout the study. However, even if intramuscular levels were increased, we were unable to demonstrate any significant effects from the postexercise creatine ingestion. Therefore, when supplementing acutely (i.e. pre- and/or post-exercise) dosage amount (i.e. a higher dosage) could be an important determinant of creatine's effects within the muscle. Unlike protein supplementation where a series of very well designed studies by Stuart Phillip's lab (Breen and Phillips 2012) and others (van Loon and Gibala 2011) have determined the threshold for protein dosage and maximal protein synthesis; to date, no similar studies have been performed for creatine supplementation. It could be suggested that a certain amount or "threshold" of creatine is needed to regulate muscle growth when taken acutely. Future work into the timing of creatine supplementation should examine different dosage amounts and its ability to acutely regulate hypertrophic signalling.

It should be noted that the $\mathrm{CHO}$ group was $\sim 6 \mathrm{~kg}$ heavier compared to the $\mathrm{CrM}-\mathrm{CHO}$ group at baseline. Although this body mass difference was not deemed significant, it could be argued that this difference may have played a role in adaptive responses to the resistance exercise program. However, total lifting volume data indicated that both groups increased their workload by similar amounts (CrM$\mathrm{CHO} \sim 20 \%$ and $\mathrm{CHO} \sim 21 \%$ ) and taken together with the lean mass changes; we can argue that the body mass difference at baseline did not significantly influence the results of the study. Another interesting observation was higher fat intakes in the CHO group when compared with the CrM$\mathrm{CHO}$ group. It is not readily apparent as to why the $\mathrm{CHO}$ group consumed higher amounts of fat. Regardless, there is little evidence to suggest that higher fat intakes would have a significant effect on the adaptations to resistance training. More importantly, no significant differences were shown in total protein intake and total calories, both known to influence muscle growth and adaptations to exercise.

To our knowledge, the current study is the first to examine long-term effects of $\mathrm{CrM}$ ingestion on serum anabolic hormones, IGF-1 and testosterone responses to resistance exercise in middle to older men. It is evident from previous literature that both hormones are important to maximize functional adaptations to resistance training (Schroeder et al. 2013). In fact, exogenous administration of testosterone to hypogonadal males has resulted in significant increases in muscle mass and decreases in fat mass as well as increases in muscle strength (Bhasin and Woodhouse 2001). In the current study, baseline serum testosterone and IGF-1 levels were close or within the normal ranges for men: 29.9-269.2 pg/ $\mathrm{ml}$, testosterone and 180-810 pg/ml, IGF-1 (Vermeulen 1996). Following the 12-week training and supplementation protocol, no significant changes in IGF-1 or testosterone levels were observed over the course of the study. However, there was a trend towards significance for higher serum
IGF-1 between 4 and 8 weeks for both groups $(p=0.066)$. The results of this study are in agreement with previous findings using resistance training only in older men (Nicklas and Ryan 1995; Brose and Parise 2003). Nicklas and colleagues (Nicklas and Ryan 1995) demonstrated that 16 weeks of progressive resistance training in males older than 55 years of age had no significant influence on baseline serum IGF1 , testosterone and growth hormone concentrations. Though serum growth hormone was increased following an acute bout of resistance exercise, serum concentrations of testosterone and IGF-1 were not affected by the resistance training program. Likewise, Izquierdo and colleagues (Izquierdo and Hakkinen 2001) showed no significant changes in serumfree testosterone following a 16-week resistance training program in middle and older aged males. Interestingly, similar to the current study, upper and lower body strength was increased over time. It could be suggested that since blood samples were obtained $48 \mathrm{~h}$ following the last bout of exercise, it is likely that serum IGF-1 and testosterone concentrations returned to baseline levels. Indeed, previous studies examining the short-term response to serum IGF-1 and testosterone concentrations following an acute bout of resistance exercise have demonstrated an increase shortly within the recovery period (12-48 h) (Florini and Ewton 1996), with levels normally returning to baseline within $48 \mathrm{~h}$. Furthermore, creatine supplementation and/or resistance exercise are potentially increasing intramuscular IGF-1 and thus enhancing the anabolic status of the cell (Singh et al. 1999; Burke et al. 2008). With this in mind, given the significant increases in lean muscle mass, total myofribillar protein content and upper and lower body strength in the current study; it could be speculated that transient increases in serum and muscle IGF-1 and/or testosterone shortly following the resistance exercise session are eliciting their anabolic effects within the muscle.

In summary, the results of the present study indicate that post-exercise supplementation with CrM combined with a 12-week high intensity resistance training program does not provide greater enhancement of body composition or muscle strength compared to $\mathrm{CHO}$ ingestion alone in middle to older males. However, it is clear that high intensity resistance training can significantly enhance body composition and muscular strength as evident by changes at the whole muscle and cellular level, and thus be an effective method at combating muscle wasting (sarcopenia) and frailty, commonly seen in the elderly. Future studies should examine the acute and chronic effects of different dosages of creatine ingestion before and/or after resistance exercise on pathways involved in skeletal muscle growth.

Acknowledgments The authors would like to thank the study participants for their hard work and willingness to donate muscle biopsy samples. This work was supported by the Baylor University Young Investigator Development Program Grant (MC). 
Conflict of interest The authors declare no conflict of interest.

Open Access This article is distributed under the terms of the Creative Commons Attribution License which permits any use, distribution, and reproduction in any medium, provided the original author(s) and the source are credited.

\section{References}

Antonio J, Ciccone V (2013) The effects of pre versus post workout supplementation of creatine monohydrate on body composition and strength. J Int Soc Sports Nutr 10(1):36. doi:10.1186/1550-2783-10-36

Bales C, Ritchie C (2002) Sarcopenia, weight loss, and nutritional frailty in the elderly. Annu Rev Nutri 22:309-323

Becque M, Lochman J (2000) Effects of oral creatine supplementation on muscular strength and body composition. Med Sci Sports Exerc 32(3):654-658

Bemben M, Witten M, Carter J, Eliot K, Knehans A, Bemben D (2010) The effects of supplementation with creatine and protein on muscle strength following a traditional resistance training program in middle-aged and older men. J Nutr Health Aging 14(2): 155-159

Bermon S, Venembre P, Sachet C, Valour S, Dolisi C (1998) Effects of creatine monohydrate ingestion in sedentary and weight-trained older adults. Acta Physiol Scand 164(2):147-155

Bhasin S, Woodhouse L (2001) Proof of the effect of testosterone on skeletal muscle. J Endocrinol 170(1):27

Borst S (2004) Interventions for sarcopenia and muscle weakness in older people. Age Ageing 33(6):548-555

Bradford M (1976) A rapid and sensitive method for the quantification of microgram quantities of protein utilizing the principle of protein-dye binding. Anal Biochem 72:248-254

Breen L, Phillips SM (2012) Nutrient interaction for optimal protein anabolism in resistance exercise. Curr Opin Clin Nutr Metab Care 15(3):226-232. doi:10.1097/MCO.0b0 $13 \mathrm{e} 3283516850$

Brose A, Parise G (2003) Creatine supplementation enhances isometric strength and body composition improvements following strength exercise training in older adults. J Gerontol Biol Sci Med Sci 58:B11-B19

Buford TW, Anton SD, Judge AR, Marzetti E, Wohlgemuth SE, Carter CS, Leeuwenburgh C, Pahor M, Manini TM (2010) Models of accelerated sarcopenia: critical pieces for solving the puzzle of age-related muscle atrophy. Ageing Res Rev 9(4):369-383. doi:10.1016/j.arr.2010.04.004

Burke DG, Silver S, Holt LE, Smith Palmer T, Culligan CJ, Chilibeck PD (2000) The effect of continuous low dose creatine supplementation on force, power, and total work. Int J Sport Nutr Exerc Metab 10(3):235-244

Burke DG, Candow DG, Chilibeck PD, MacNeil LG, Roy BD, Tarnopolsky MA, Ziegenfuss T (2008) Effect of creatine supplementation and resistance-exercise training on muscle insulinlike growth factor in young adults. Int J Sport Nutr Exerc Metab 18(4):389-398

Campbell WW, Leidy HJ (2007) Dietary protein and resistance training effects on muscle and body composition in older persons. $\mathrm{J}$ Am Coll Nutr 26(6):696S-703S

Candow D, Chilibeck P (2007) Effect of creatine supplementation during resistance training on muscle accretion in the elderly. $\mathbf{J}$ Nutr Health Aging 11(2):185-188

Candow DG, Chilibeck PD (2008) Timing of creatine or protein supplementation and resistance training in the elderly. Appl Physiol
Nutr Metab (Physiologie appliquee, nutrition et metabolisme) 33(1):184-190. doi:10.1139/H07-139

Candow DG, Little JP, Chilibeck PD, Abeysekara S, Zello GA, Kazachkov M, Cornish SM, Yu PH (2008) Low-dose creatine combined with protein during resistance training in older men. Med Sci Sports Exerc 40(9):1645-1652. doi:10.1249/MSS.0b01 3e $318176 \mathrm{~b} 310$

Casey A, Constantin-Teodosiu D (1996) Creatine ingestion favorably affects performance and muscle metabolism during maximal exercise in humans. Am $\mathbf{J}$ Physiol Endocrinol Metab 271(1):E31-E37

Chilibeck PD, Stride D, Farthing JP, Burke DG (2004) Effect of creatine ingestion after exercise on muscle thickness in males and females. Med Sci Sports Exerc 36(10):1781-1788

Chrusch M, Chilibeck P, Chad K, Davison K, Burke D (2001) Creatine supplementation combined with resistance training in older men. Med Sci Sports 33(12):2111-2117

Cooke MB, La Bounty P, Buford T, Shelmadine B, Redd L, Hudson G, Willoughby DS (2011) Ingestion of 10 grams of whey protein prior to a single bout of resistance exercise does not augment Akt/mTOR pathway signaling compared to carbohydrate. J Int Soc Sports Nutr 8:18. doi:10.1186/1550-2783-8-18

Cribb P, Hayes A (2006) Effects of supplement timing and resistance exercise on skeletal muscle hypertrophy. Med Sci Sports Exerc 38(11):1918-1925

Doherty T (2003) Invited review: aging and sarcopenia. J Appl Physiol 95(4):1717-1727

Eijnde BO, Van Leemputte M, Goris M, Labarque V, Taes Y, Verbessem P, Vanhees L, Ramaekers M, Vanden Eynde B, Van Schuylenbergh R, Dom R, Richter EA, Hespel P (2003) Effects of creatine supplementation and exercise training on fitness in men 55-75 years old. J Appl Physiol (1985) 95(2):818-828. doi:1 0.1152/japplphysiol.00891.2002

Elliot K, Knehans A, Bemben D, Witten M, Carter J, Bemben M (2008) The effects of creatine and whey protein supplementation on body composition in men aged 48-72 years during resistance training. J Nutr Health Aging 12(3):208-212

Florini J, Ewton D (1996) Growth hormone and insulin-like growth factor system in myogenesis. Endocr Rev 17(5):481-517

Gotshalk L, Kraemer W, Mendonca M, Vingren J, Kenny A, Spiering B, Hartfield D, Fragala MV, Volek JS (2007) Creatine supplementation improves muscular performance in older women. Eur J Appl Physiol 102(2):223-231

Hunter G, MCarthy J, Bamman M (2004) Effects of resistance training on older adults. Sports Med 34(5):329-348

Izquierdo M, Hakkinen K (2001) Effects of strength training on muscle power and serum hormones in middle-aged and older men. J Appl Physiol 90(4):1497-1507

Jager R, Harris RC, Purpura M, Francaux M (2007) Comparison of new forms of creatine in raising plasma creatine levels. J Int Soc Sports Nutr 4:17. doi:10.1186/1550-2783-4-17

Janssen I, Heymsfield S, Ross R (2002) Low relative skeletal muscle mass (sarcopenia) in older persons is associated with functional impairment and physical ability. J Am Geriatr Soc 50(5):889-896

Kreider R (2003) Effects of creatine supplementation on performance training adaptations. Mol Cell Biochem 244(1-2):89-94

Nicklas B, Ryan A (1995) Testosterone, growth hormone and IGF-1 responses to acute and chronic resistive exercise in men aged 5570. Int J Sports Med 16(7):445-450

Olsen S, Aagaard P, Kadi F, Tufekovic G, Verney J, Olesen J, Suetta C, Kjaer M (2006) Creatine supplementation augments the increase in satellite cell and Myonuclei number in human skeletal muscle induced by strength training. J Physiol 573(2):525-534

Rawson E, Volek J (2003) Effects of creatine supplementation and resistance training on muscle strength and weightlifting 
performance. J Strength Cond Res/Natl Strength Cond Assoc 17(4):822-831

Rawson E, Wehnert ML, Clarkson PM (1999) Effects of 30 days of creatine ingestion in older men. Eur J Appl Physiol Occup Physiol 80(2):139-144

Schroeder ET, Villanueva M, West DD, Phillips SM (2013) Are acute post-resistance exercise increases in testosterone, growth hormone, and IGF-1 necessary to stimulate skeletal muscle anabolism and hypertrophy? Med Sci Sports Exerc 45(11):2044-2051. doi:10.1249/MSS.0000000000000147

Singh MA, Ding W, Manfredi TJ, Solares GS, O’Neill EF, Clements KM, Ryan ND, Kehayias JJ, Fielding RA, Evans WJ (1999) Insulin-like growth factor I in skeletal muscle after weight-lifting exercise in frail elders. Am J Physiol 277(1 Pt 1):E135-E143

Snow R, McKenna M (1998) Effect of creatine supplementation on sprint exercise performance and muscle metabolism. J Appl Physiol 84(5):1667-1673
Stark M, Lukaszuk J, Prawitz A, Salacinski A (2012) Protein timing and its effects on muscular hypertrophy and strength in individuals engaged in weight-training. J Int Soc Sports Nutr 9(1):54. doi:10.1186/1550-2783-9-54

van Loon LJ, Gibala MJ (2011) Dietary protein to support muscle hypertrophy. Nestle Nutr Inst Workshop Ser 69:79-89. doi:10.1159/000329287 (discussion 89-95)

Vermeulen A (1996) Decreased androgen levels and obesity in men. Ann Med 28(1):13-15

Williams M, Branch J (1998) Creatine supplementation and exercise performance: an update. J Am Coll Nutr 17(3):216-234

Willoughby D, Rosene J (2001) Effects of oral creatine and resistance training on myosin heavy chain expression. Med Sci Sports Exerc $33: 1674-1681$ 